\title{
Effect of Elevated Intra-Abdominal Pressure on Portal Vein and Superior Mesenteric Artery Blood Flow in a Rat
}

\author{
Jorge Mogilner, MD, ${ }^{1}$ Igor Sukhotnik, MD, ${ }^{1}$ Vera Brod, PhD, ${ }^{3}$ Lili Hayari, MD, ${ }^{2}$ Arnold G. Coran, MD, ${ }^{4}$ \\ Eitan Shiloni, MD, ${ }^{3}$ Samuel Eldar, MD, ${ }^{1}$ and Haim Bitterman, $\mathrm{MD}^{3}$
}

\begin{abstract}
Aim: Recent clinical experience suggests that minimal access portoenterostomy (the Kasai procedure) for biliary atresia leads to transplantation sooner, compared to the traditional open approach. It should be emphasized that elevated intra-abdominal pressure (IAP) may reduce hepatic and portal blood flow and thus may cause histologic liver damage. The aim of the present study was to evaluate the effects of IAP on blood flow in the portal vein (PV), compared to the superior mesenteric artery (SMA), and on the systemic mean arterial blood pressure (MABP).

Materials and Methods: Male Sprague-Dawley rats were anesthetized with intraperitoneal ketamine $(90 \mathrm{mg}$ per $\mathrm{kg}$ ) and xylasine (13 mg per $\mathrm{kg}$ ). Polyethylene catheters (PE-50) were introduced into the right carotid artery for the measurement of MABP. After a midline laparotomy, the SMA and PV were isolated. Ultrasonic bloodflow probes were placed on the vessels for the continuous measurement of regional blood flow. Two large-caliber percutaneous peripheral intravenous catheters were introduced into the peritoneal cavity for inflation of air and for the measurement of IAP. The time course of MABP and SMA and PV flow as well as the relationship between IAP and SMA and PV flow were determined.

Results: Although all three hemodynamic parameters decreased with the increase in the IAP, the most significant changes were observed in PV blood flow. IAP at $3 \mathrm{~mm} \mathrm{Hg}$ resulted in a $26 \%$ decrease in PV flow $(P<$ $0.05)$, a $19 \%$ decrease in SMA flow $(P<0.05)$, and an $11 \%$ decrease in MABP $(P<0.05)$. IAP at $6 \mathrm{~mm} \mathrm{Hg}$ caused a two-fold decrease in PV flow $(P<0.05)$, a $30 \%$ decrease in SMA flow $(P<0.05)$, and a $19 \%$ decrease in MABP $(P<0.05)$. There were no changes in the time course of MABP and PV and SMA flow. PV and SMA flow returned to normal values immediately after abdominal deflation.

Conclusions: Persistent IAP decreased MABP, SMA, and, especially, PV flow by 50\%. We speculate that in biliary atresia patients with already present liver dysfunction, decrease in SMA flow and even a greater decrease in PV flow from increased IAP, which occurs during a laparoscopic Kasai procedure, may further compromise liver function. This may be one of the explanations for the progression to earlier transplantation in infants undergoing a laparoscopic Kasai procedure.
\end{abstract}

\section{Introduction}

$\mathbf{T}$ HE SURGICAL MANAGEMENT of biliary atresia is still a great challenge for pediatric surgeons. Since Kasai introduced the hepatic portoenterostomy for surgical relief of biliary obstruction in infants in 1957, ${ }^{1}$ this procedure has revolutionized the surgical management of biliary atresia and has become the standard of care for initial management of this disorder. The Kasai operation is traditionally performed through a wide, painful, muscle-cutting laparotomy that quite often is followed by pain and peritoneal adhesions, which may cause difficulty in performing a subsequent liver transplantation. Advances in minimally invasive surgery have allowed the portoenterostomy to be approached by using these techniques. The laparoscopy was first applied to neonatal jaundice as a diagnostic tool. Laparoscopic cholecystocholangiography has been introduced as an alternative procedure to avoid unnecessary laparotomy. ${ }^{2}$ In more recent

${ }^{1}$ Department of Pediatric Surgery, Bnai Zion Medical Center, ${ }^{2}$ Department of Pediatric Surgery, Rambam Medical Center, and ${ }^{3}$ Department of Medicine, Ischemia-Shock Research Laboratory, Carmel Medical Center; The Ruth and Bruce Rappaport Faculty of Medicine, Technion-Israel Institute of Technology, Haifa, Israel.

${ }^{4}$ Section of Pediatric Surgery, Mott Children's Hospital, University of Michigan, Ann Arbor, Michigan.

JM and IS contributed equally to the preparation of this manuscript. 
years, the introduction of minimal access portoenterostomy, with its potential for excellent visualization of the portal structures, has added a new dimension to the minimal access surgical approach to this disorder. ${ }^{3}$ This procedure can avoid or decrease the postoperative complications, such as pain, breathing difficulty, or adhesion formation, and improves cosmesis. ${ }^{4}$ Besides all the advantages of minimally invasive access, it allows excellent visibility and dissection of tiny hilar structures. ${ }^{5}$ Potential disadvantages of the laparoscopic approach include only two-dimensional optics and limitations in degrees of movement with the laparoscope movement. ${ }^{6}$ In the last few years, European surgeons have suggested, at the IPEG meeting in 2007, that minimal access portoenterostomy leads to transplantation sooner, and with a higher rate, compared to the traditional open approach. ${ }^{7}$ The causes of this fact remain unclear. In this regard, it should be emphasized that elevated intra-abdominal pressure (IAP) may adversely affect the barrier and metabolic functions of the small intestine, may reduce hepatic and portal blood flow, and thus may cause histologic liver damage. The aim of the present study was to evaluate the effects of IAP on blood flow in the portal vein (PV), superior mesenteric artery (SMA), and on the systemic mean arterial blood pressure $(\mathrm{MABP})$ in a rat model.

\section{Materials and Methods}

\section{Animals}

The experimental protocol was approved by the Guide for the Care and Use of Laboratory Animals, Rappaport Faculty of Medicine, Technion (Haifa, Israel). Male Sprague-Dawley rats, weighing 280-300 g, were housed in individual stainless steel cages under standardized conditions (12-hour lightdark cycle, controlled room temperature) for 5-7 days. The rats had free access to water and were pair fed with standard chow.

\section{Experimental design}

The animals were anesthetized with intraperitoneal ketamine (90 mg per $\mathrm{kg}$ ) and xylasine (13 mg per $\mathrm{kg}$ ). Rectal temperature was monitored continuously (Yellow Springs Instruments, Letchworth, UK) and maintained within normal limits throughout the experiments by a heating blanket. The trachea was exposed and cannulated with a polyethylene catheter (PE-240) to ensure an open airway. Animals breathed spontaneously. Polyethylene catheter (PE-50) was introduced into the right carotid artery for measurements of blood pressure. Arterial pressure was recorded by using calibrated pressure transducers. After a midline laparotomy, the SMA and PV were isolated. Ultrasonic blood-flow probes (Transonic, Ithaca, NY) were placed on the vessels for the continuous measurement of regional blood flow to the small bowel and liver. Because of the complex instrumentation required in these experiments and the prolonged surgery required for the installation of the cannulas and probes, only one vascular bed was evaluated in each animal. Two large-caliber percutaneous peripheral intravenous catheters were introduced into the peritoneal cavity for the inflation of air and for the measurement of IAP, and the abdominal cavity was then closed. One IAP catheter was connected to a multichannel recorder (CyberAmp 380; Axon Instruments, Foster City, CA), using PDCR75 pressure transducers (Cobe CDX III; Argon, Athens,
TX), and intra-abdominal pressure was recorded. Air was slowly insufflated until IAP reached $6 \mathrm{~mm} \mathrm{Hg}$. During the first hour, the relationship between IAP and MABP, SMA, and PV blood flow was evaluated. For this reason, after abdominal insufflation until IAP reached $6 \mathrm{~mm} \mathrm{Hg}$, gas was gradually removed until the IAP reached $0 \mathrm{~mm} \mathrm{Hg}$, and the relationship between IAP and systemic blood pressure, and SMA and PV blood flow, was determined. During the next 2 hours, gas was either added or removed to keep the IAP constant at $6 \mathrm{~mm} \mathrm{Hg}$. The time course of MABP and SMA and PV flow was determined. At the end of the experiments, the rats were sacrificed by open pneumothorax.

\section{Statistical analysis}

Data are presented as the mean \pm one standard error. MABP is expressed in $\mathrm{mm} \mathrm{Hg}$. Multivariate analysis of variance with repeated measures was used to determine if a variable changed significantly with respect to time. Comparisons between mean values and initial value were done by the $t$ test.

\section{Results}

Figure 1 illustrates the relationship between MABP and SMA and portal vein blood flow and IAP. As expected, IAP at $6 \mathrm{~mm} \mathrm{Hg}$ caused a $19 \%$ decrease in MABP $(P<0.05)$ with a subsequent gradual increase when the abdomen was gradually decompressed to values not significantly different from those before abdominal insufflations. An IAP of $3 \mathrm{~mm} \mathrm{Hg}$ resulted in an $11 \%$ decrease in MABP $(P<0.05)$, compared to the initial value. Abdominal insufflations at $6 \mathrm{~mm} \mathrm{Hg}$ induced a marked decrease in SMA (30\% of baseline; $P<0.05)$, and an IAP at $3 \mathrm{~mm} \mathrm{Hg}$ induced a 19\% decrease in SMA blood flow, compared to baseline levels $(P<0.05)$. Later, the gradual decrease in IAP was followed by a parallel gradual increase in SMA blood flow, reaching values that were not significantly different from initial levels at the end of the experiment. As also shown in Figure 1, the changes in PV blood flow were more significant than those in MABP and SMA blood flow. IAP at $6 \mathrm{~mm} \mathrm{Hg}$ resulted in a two-fold decrease in PV blood flow $(5.8 \pm 1.9$ vs. $10.8 \pm 1.1 \mathrm{~mL} / \mathrm{min} ; P<0.05)$, while IAP at $3 \mathrm{~mm} \mathrm{Hg}$ caused a $26 \%$ decrease in PV flow $(P<0.05)$, compared to initial levels. These changes in PV blood flow also resolved gradually upon decrease in IAP, reaching values that were not significantly different from baseline.

The time-related changes in SMA and PV blood flow and in MABP are shown in Figure 2 throughout an interval of 2 hours. During this time, gas was either added or removed to keep the IAP constant at $6 \mathrm{~mm} \mathrm{Hg}$. The time course, of MABP and SMA and PV flow was determined. As demonstrated in Figure 2, all three hemodynamic parameters showed a trend toward gradual decrease over time, but did not fall below $95 \%$ of baseline values during 2 hours of observation.

\section{Discussion}

Abdominal compartment syndrome (ACS) is defined as increased intra-abdominal pressure leading to adverse homodynamic and respiratory effects. Since Kron et al. in the early 1980s described increased IAP in 11 patients following aortic aneurysm surgery, which leads to oliguria and death, 8 ACS became a commonly recognized entity and is well described in various clinical situations. The most common of 


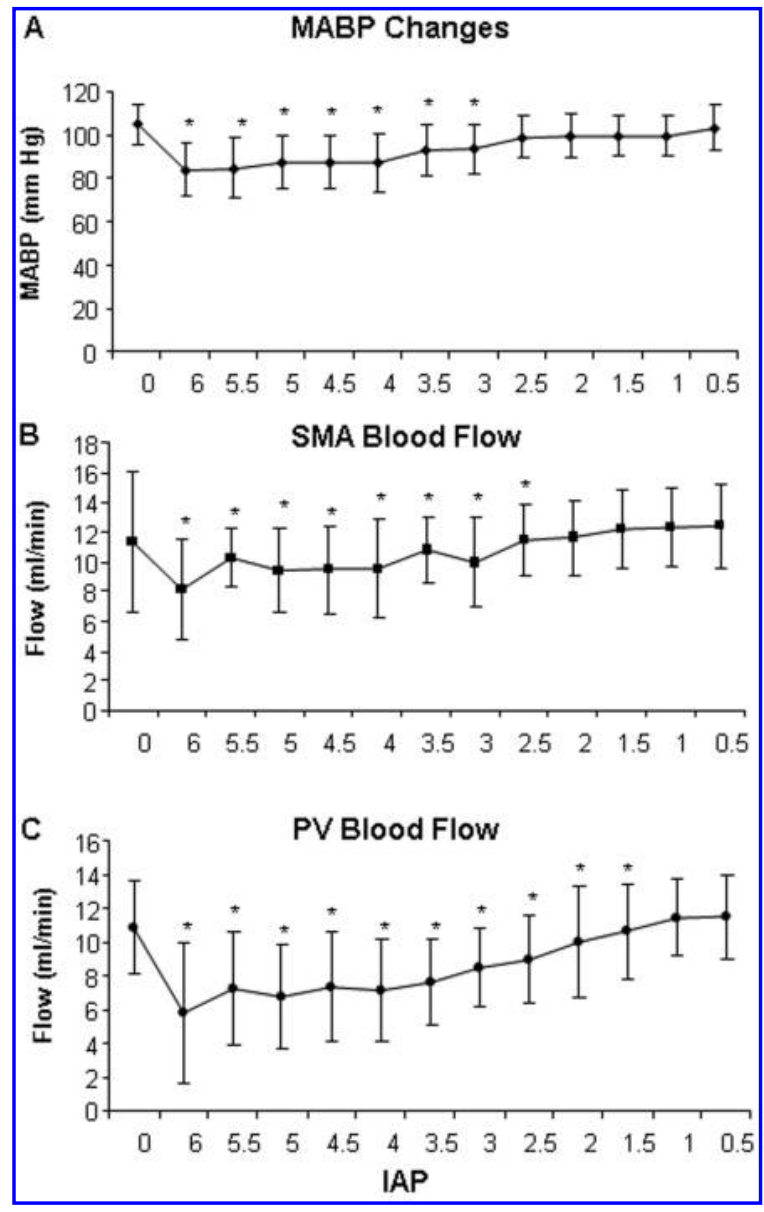

FIG. 1. Correlation between directly measured intra-abdominal pressure and mean arterial blood pressure (A), blood flow in superior mesenteric artery (B), and in the portal vein $(\mathbf{C})$. Values are mean \pm standard deviation. IAP, intra-abdominal pressure; MABP, mean arterial blood pressure; SMA, superior mesenteric artery; $\mathrm{PV}$, portal vein. ${ }^{*} \mathrm{P}<$ 0.05 versus baseline levels.

these are intra-abdominal or retroperitoneal hemorrhage, visceral edema, pancreatitis, acute gastric or bowel dilation, mesenteric venous obstruction, abdominal packs, tense ascites, peritonitis, reduction of large hernias, large abdominal or retroperitoneal tumors, and so on. ${ }^{9,10}$

Although the carbon dioxide $\left(\mathrm{CO}_{2}\right)$ pneumoperitoneum during laparoscopic operations does not exceed $15 \mathrm{~mm} \mathrm{Hg}$ (ACS can develope when IAP is greater than $20 \mathrm{~mm} \mathrm{Hg}$ ), many clinical reports have demonstrated that laparoscopy can have adverse effects on cardiovascular, pulmonary, and renal function. ${ }^{11,12}$ In the present study, we evaluated the consequences of increased intra-abdominal pressure on PV blood flow, compared to blood flow in the SMA, and changes in systemic blood pressure, because of its potential relationship with liver damage previously reported following prolonged laparoscopic procedures. It is true that clinical laparoscopy generally requires lower IAP levels than were used in some animal studies. ${ }^{11,13}$ Therefore, in the current study, the maximal level of $6 \mathrm{~mm} \mathrm{Hg}$ was selected for the IAP.

Our data show that elevated IAP resulted in a mild decrease in systemic blood pressure. IAP at $3 \mathrm{~mm} \mathrm{Hg}$ resulted in an $11 \%$ decrease in MABP, while IAP at $6 \mathrm{~mm} \mathrm{Hg}$ caused a $19 \%$ decrease in MABP. This is in keeping with other investigators who found a mild decrease in blood pressure, even at very high levels of IAP. ${ }^{13}$ It is well known that elevation in IAP leads to a reduction in cardiac output. ${ }^{11,13,14}$ The diminished cardiac output results from decreased inferior vena caval flow secondary to direct compression of the inferior vena cava as well as from increased thoracic pressure, which decreases both inferior and superior vena caval flow. The increased thoracic pressure also leads to cardiac compression with decreased ventricular end-diastolic volumes. ${ }^{15}$

Intestinal ischemia and infarction have been described during prolonged laparoscopy despite apparently normal hemodynamic and renal function. ${ }^{16,17}$ The mechanisms of reduced splanchnic circulation in ACS involve decreased cardiac output, increased SMA vascular resistance due to vascular compression, and direct parenchymal compression. Our data also suggest that elevated IAP results in a significant decrease in SMA blood flow. IAP at $3 \mathrm{~mm} \mathrm{Hg}$ induced a $19 \%$ decrease in SMA blood flow, while IAP at $6 \mathrm{~mm} \mathrm{Hg}$ resulted in a $30 \%$ decrease in SMA blood flow, compared to

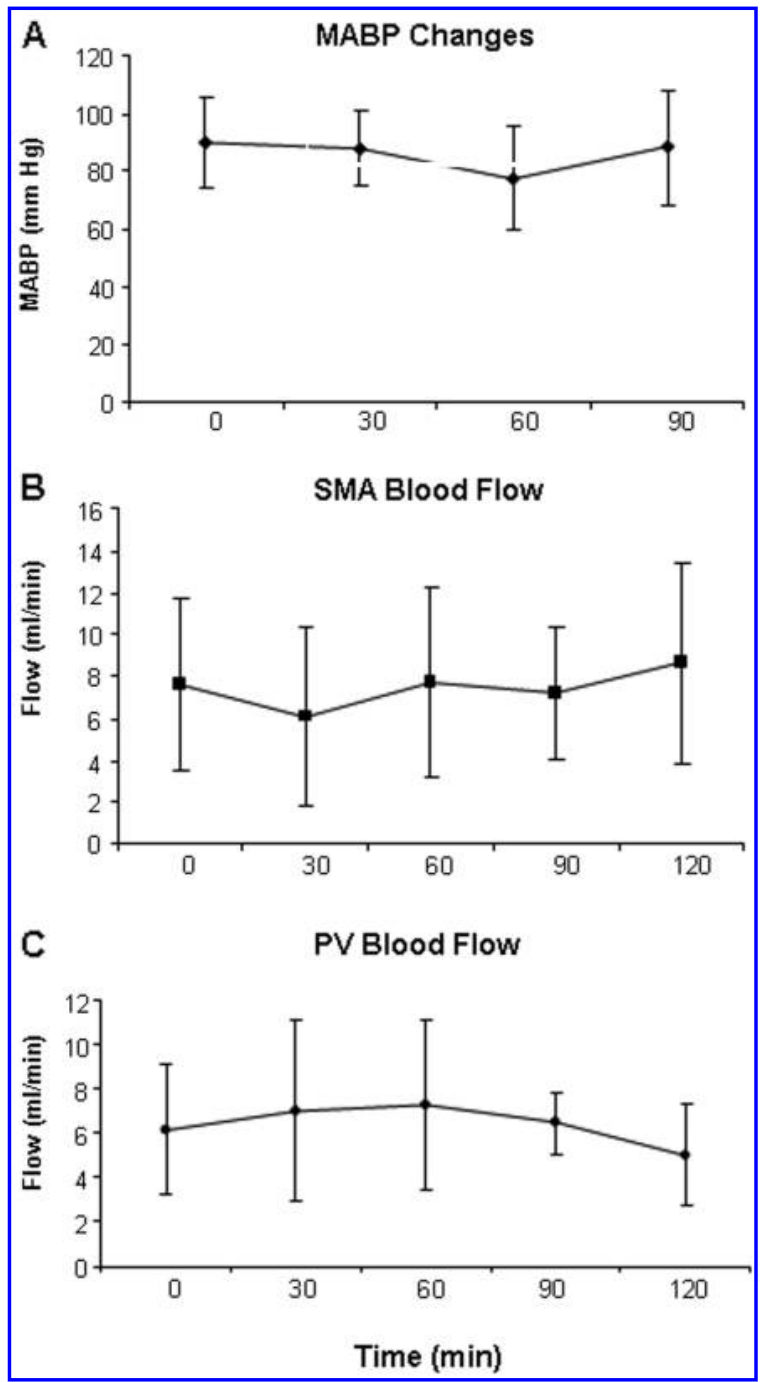

FIG. 2. Time dependence of hemodynamic variables. Values are the mean \pm standard deviation. IAP, intra-abdominal pressure; MABP, mean arterial blood pressure; SMA, superior mesenteric artery; PV, portal vein. 
baseline levels. Caldwell and Ricotta have shown that elevated IAP causes a decrease in organ blood flow in all splanchnic organs except the renal cortex and the adrenal glands. These organ blood-flow changes were greater than could be explained by the corresponding decrease in cardiac output alone. The investigators hypothesized that local control mechanisms could have been responsible for changes in organ blood flow. ${ }^{18}$

Although all three hemodynamic parameters decreased with the increase in IAP in the current study, the most significant changes were observed in PV blood flow. Increasing IAP caused a progressive decline in PV blood flow, which showed a one fourth decrease at an IAP of $3 \mathrm{~mm} \mathrm{Hg}$ and a two-fold decrease at an IAP of $6 \mathrm{~mm} \mathrm{Hg}$. The effect of elevated IAP on venous blood flow in the abdominal cavity depends on the type of vein and the degree of IAP elevation. In the human, IAP of $10-15 \mathrm{~mm} \mathrm{Hg}$ may enhance venous return by the mobilization of blood from capacitance vessels within the abdomen. ${ }^{19}$ Venous return has been shown to be impaired at an IAP as low as $15 \mathrm{~mm} \mathrm{Hg}$, decreasing with further increases in IAP. This results from increased venous resistance within the abdomen and thorax, leading to reduced caval and retroperitoneal venous flow. ${ }^{13}$ Our observation of a marked decrease in PV blood flow is in concert with that of previous investigators. In a pig model of ACS, Diebel and coworkers have found that mesenteric and intestinal mucosal flow reductions first occurred at an IAP of $20 \mathrm{~mm} \mathrm{Hg}$, while hepatic and portal flow became compromised at only $10 \mathrm{~mm} \mathrm{Hg}{ }^{20}$ The investigators concluded that modest increases in IAP can cause significant impairment of hepatic perfusion, despite a normal blood pressure and cardiac output. Rasmussen and colleagues have shown in pigs that an IAP of $25 \mathrm{~mm} \mathrm{Hg}$ results in a $66 \%$ decrease in portal venous blood flow and a 6.5-fold increase in portal/hepatic vascular resistance, compared to baseline levels; the increase in gastrointestinal vascular resistance was less pronounced in this experiment. ${ }^{21}$

\section{Conclusions}

In conclusion, this experimental study has demonstrated a marked reduction in PV flow in response to a modest elevation of IAP. The extent of the decrease in PV flow is influenced mostly by the degree of increased IAP and is less influenced by the duration of the elevated IAP. Further studies evaluating the effects of increased IAP on hepatocyte proliferation and apoptosis may shed further light on the mechanisms involved in the resultant hepatic dysfunction from increased IAP. Finally, these studies may provide some insight into the clinical observation that the laparoscopic portoenterostomy leads to an earlier, more frequent need for liver transplantation in babies with biliary atresia than does the open procedure.

\section{Disclosure Statement}

No competing financial interests exist.

\section{References}

1. Kasai M. Surgical treatment of biliary atresia. J Pediatr Surg 1974;6:5-8.

2. Nose $\mathrm{S}$, Hasegawa T, Soh H, et al. Laparoscopic cholecystocholangiography as an effective alternative exploratory laparotomy for the differentiation of biliary atresia. Surg Today 2005;35:925-928.
3. Lima M, Gargano T, De Biagi L, et al. Video-assisted treatment for biliary atresia. Pediatr Med Chir 2007;29:212-217.

4. Lee H, Hirose S, Bratton B, Farmer D. Initial experience with complex laparoscopic biliary surgery in children: Biliary atresia and choledochal cyst. J Pediatr Surg 2004;39:804-807.

5. Esteves E, Neto CE, Neto OM, et al. Laparoscopic Kasai portoenterostomy for biliary atresia. Pediatr Surg Int 2002;18: 737-740.

6. Dutta S, Woo R, Albanese CT. Minimal access portoenterostomy: Advantages and disadvanteges of standard laparoscopic and robotic techniques. J Laparoendosc Adv Surg Tech 2007;17:258-264.

7. IPEG 2007 Meeting. Oral abstracts. J Laparoendosc Adv Surg Tech 2007;17:572-579.

8. Kron IL, Harman PK, Nolan SP. The measurement of intraabdominal pressure as a criterion for abdominal re-exploration. Ann Surg 1984;199:28-30.

9. Burch JM, Moore EE, Moore FA, et al. The abdominal compartment syndrome. Surg Clin North Am 1996;76:833-842.

10. Saggi BH, Sugerman HJ, Ivatury RR, et al. Abdominal compartment syndrome. J Trauma 1998;45:597-560.

11. Winndberger U, Siegl H, Feguson JG, et al. Hemodyunamic effects of prolonged abdominal insufflations for laparoscopic procedures. Gastrointest Endosc 1995;41:121-129.

12. Kirsh AJ, Hensle TW, Chang DT, et al. Renal effects of CO2 insufflation: Oliguria and acute renal dysfunction in a rat preumoperitoneum model. Urology 1994;43:453-459.

13. Kashtan J, Green JF, Parsons EQ, et al. Hemodynamic effects of increased abdominal pressure. J Surg Res 1981;30:249-255.

14. Barnes GE, Laine GA, Giam PY, et al. Cardiovascular responses to elevation of intra-abdominal hydrostatic pressure. Am J Physiol 1985;248:R208-R213.

15. Saggi BH, Sugerman HJ, Ivatury RR, et al. Abdominal compartment syndrome. J Trauma 1998:45:597-609.

16. Paul A, Troidl H, Peters $S$, et al. Fatal intestinal ischemia following laparoscopic cholecystectomy. Br J Surg 1994;81: 1207-1211.

17. Jaffe V, Russel RC. Fatal intestinal ischemia following laparoscopic cholecystectomy. Br J Surg 1994;81:1927-1929.

18. Caldwell CB, Ricotta JJ. Changes in visceral blood flow with elevated intra-abdominal pressure. I Surg Res 1987;43:14-20.

19. Kelman GR, Swapp GH, Smith I, et al. Cardiac output and arterial blood-gas tension during laparoscopy. $\mathrm{Br}$ J Anaest 1972;44:1155-1161.

20. Diebel LN, Wilson RF, Dulchavsky SA, et al. Effect of increased intra-abdominal pressure on hepatic arterial portal venous and hepatic microcirculatory blood flow. I Trauma 1992;33:279-282.

21. Rasmussen IB, Berggren U, Arvidsson D, et al. Effects of pneumoperitoneum on splanchnic hemodynamics: An experimental study in pigs. Eur J Surg 1995;161:819-826.

Address reprint requests to: Jorge Mogilner, MD

Department of Pediatric Surgery, Bnai Zion Medical Center The Ruth and Bruce Rappaport Faculty of Medicine Technion-Israel Institute of Technology

47 Golomb Street

P.O.B. 4940

Haifa, 31048

Israel

E-mail: olguer@bezeqint.net 